\title{
A comparison of the safety and efficacy of polyethylene glycol 4000 and lactulose for the treatment of constipation in pregnant women: a randomized controlled clinical study
}

\author{
Hang Li, Pingfang Zhang, Yuzheng Xue \\ Department of Gastroenterology, Affiliated Hospital of Jiangnan University, Wuxi, China \\ Contributions: (I) Conception and design: H Li; (II) Administrative support: Y Xue; (III) Provision of study materials or patients: H Li; (IV) Collection \\ and assembly of data: P Zhang; (V) Data analysis and interpretation: H Li; (VI) Manuscript writing: All authors; (VII) Final approval of manuscript: \\ All authors. \\ Correspondence to: Yuzheng Xue. Department of Gastroenterology, Affiliated Hospital of Jiangnan University, Wuxi 214000 , China. \\ Email: xueyz001@163.com.
}

Background: Constipation is a common gastrointestinal complication during pregnancy. The prevalence
of constipation is higher in pregnant women compared to the general population owing to the physiological
changes that occur throughout pregnancy. Lactulose and polyethylene glycol belong to a class of medications
known as osmotic laxatives. The purpose of this study is to compare the safety and efficacy of polyethylene
glycol and lactulose in pregnant women with constipation.

Methods: In this study, we selected 113 pregnant women with constipation who attended the Department of Gastroenterology, Affiliated Hospital of Jiangnan University from May 1, 2017 to April 30, 2020. The included patients were randomly divided into two groups. The observation group used polyethylene glycol 4000 (manufactured by Beau four Ipsen Industry, France, National Medicine Standard: H20130145), $10 \mathrm{~g}$, twice daily. The control group used lactulose (manufactured by Abbott Biologicals BV, Netherlands, National Medicine Standard: H20120387) $15 \mathrm{~mL}$, twice daily. Both groups were given a 3-week course of treatment, and were observed for any adverse drug reactions. The clinical effects were compared every week during the 3-week treatment.

Results: Treatment was found to be effective in both the observation and control groups, as measured by a significant difference in the Wexner constipation scores of patients before and after treatment $(\mathrm{P}<0.05)$. Although no significant differences in the final effect of treatment were observed between the two groups $(\mathrm{P}>0.05)$, analysis of the Wexner scores at the first and second week of treatment indicated that polyethylene glycol had a faster therapeutic effect than lactulose $(\mathrm{P}=0.06, \mathrm{P}=0.029)$.

Conclusions: Compared with lactulose, Polyethylene glycol 4000 can significantly shorten the treatment course for constipation during pregnancy.

Keywords: Pregnant; constipation; polyethylene glycol; lactulose; randomized control

Submitted Jul 31, 2020. Accepted for publication Sep 25, 2020.

doi: 10.21037/apm-20-1674

View this article at: http://dx.doi.org/10.21037/apm-20-1674

\section{Introduction}

Constipation is a common gastrointestinal complication during pregnancy. The prevalence of constipation is higher in pregnant women compared to the general population, particularly due to the physiological changes that occur throughout pregnancy. Constipation reportedly affects between $2.6 \%$ and $24.8 \%$ of Asians (1) and $40 \%$ of pregnant women (2). The prevalence and severity of constipation also varies depending on the stage of the pregnancy. The occurrence of constipation in early pregnancy is brought 
on by hormonal factors and mechanical changes related to pregnancy progression. Previous studies have shown that female sex hormones affect gastrointestinal motility in non-pregnant women (3). For instance, progesterone and somatostatin may inhibit the release of motilin, a peptide hormone that stimulates gastrointestinal motility (4). Also, relaxant is a polypeptide that prevents smooth muscle contraction during pregnancy, and also inhibits smooth muscle activity in the gastrointestinal tract (5). Moreover, estrogen and progesterone induce increased secretion of renin, which converts angiotensinogen into angiotensin-I. This is then converted into angiotensin-II by the angiotensin converting enzyme (ACE), which ultimately leads to increased levels of aldosterone during pregnancy. Comprehensive studies on colon perfusion have shown that aldosterone increases water absorption during pregnancy, especially in the second trimester (2).

Constipation during pregnancy may also potentially affect the growth and development of the fetus. Forced defecation during pregnancy can cause serious adverse consequences and even lead to miscarriage or premature delivery, and postpartum constipation is not conducive to wound healing. The treatment of constipation typically depends on its severity and duration. First-line treatments include changes in diet, lifestyle, exercise, and use of laxatives. Considering the safety implications for the mother and child, treatment of constipation during pregnancy requires special attention (6).

If the short-term symptoms of constipation are not relieved through lifestyle adjustment, laxatives can be prescribed as appropriate. Laxatives may help to reduce the occurrence of constipation, avoid premature birth, and prevent intestinal obstruction, hemorrhoids, and other anorectal diseases, thus improving quality of life. The efficacy, safety, drug dependence, and potency ratio should be considered when prescribing laxatives. Due to the particularity of pregnancy and breastfeeding, the choice of laxatives in pregnant women should be based on ensuring the safety of the mother and the fetus $(7,8)$. The ideal laxative should exhibit good curative effect and tolerability, and should not be absorbed into the blood (no teratogenic effect) or transferred into breast milk.

Lactulose is an artificially synthesized disaccharide, and belongs to a class of medications known as osmotic laxatives. In China, lactulose is commonly prescribed to treat constipation during pregnancy and in the postpartum period. The United States Food and Drug Administration (FDA) has also approved it for this purpose. Lactulose is a prebiotic, recognized by the World Gastroenterology Organization (WGO), and is thus not digested in the small intestine. In the colon, it causes an osmotic gradient, and under the action of osmotic pressure, promotes softening of the stool, thereby making it easier to pass. Lactulose can also increase the number of intestinal probiotics such as Lactobacillus and Bifidobacterium, reduce the number of anaerobic bacteria, and regulate the micro-ecological balance of the intestinal tract (9). Through the action of bacteria in the colon, lactulose can be converted into acetic acid and lactic acid, resulting in an increase in colonic acidity and the promotion of peristalsis, ultimately assisting in the excretion of feces (9).

Lactulose can significantly improve average stool characteristics and there are no serious adverse reactions following treatment. Furthermore, treatment efficiency and satisfaction rates are high (10-12) Lactulose is also used postpartum, and significantly improves bowel motility, stool characteristics, and shortens hospitalization time. Applied to patients with postpartum perineal lacerations, it significantly improves first bowel movement pain and also shortens hospitalization time $(13,14)$. In terms of safety, since lactulose is not absorbed into the blood, it will not cause blood sugar fluctuations and does not affect nutrient absorption, fetal development, or breastfeeding (15).

Polyethylene glycol is a long-chain high molecular weight polymer (relative molecular mass exceeds 3,000). Due to a lack of enzymes that degrade polyethylene glycol, it cannot be absorbed in the intestines and is not decomposed by intestinal bacteria. Polyethylene glycol increases the water content of the stool, thereby softening the stool and making it easier to excrete. However, the stool volume does not increase significantly. Like lactulose, polyethylene glycol is also an osmotic laxative, however polyethylene glycol does not affect colonic transit time or motility (16). Previous controlled clinical studies have shown that polyethylene glycol 3350/4000 can significantly improve the number of bowel movements and stool characteristics in patients with chronic functional constipation, and its efficacy has been shown to be better than both lactulose and psyllium hydrocolloid $(17,18)$. And PEG is found to have better efficacy than lactulose, can treat chronic constipation in young children, and is well tolerated (19).

To our knowledge, there have been no randomized clinical trials comparing polyethylene glycol and lactulose for the treatment of constipation during pregnancy. Therefore, considering the higher incidence of constipation among pregnant women and the serious potential 
complications, as well as the existing evidence about the effect of osmotic laxatives on the human digestive system, this controlled randomized clinical trial was performed. We present the following article in accordance with the CONSORT reporting checklist (available at http://dx.doi. org/10.21037/apm-20-1674).

\section{Methods}

\section{Setting and study design}

Based on the Rome IV diagnostic criteria for chronic functional constipation (18), we selected a total of 113 pregnant women with constipation who were admitted to the Department of Gastroenterology at the Affiliated Hospital of Jiangnan University from May 1, 2017 to April 30, 2020. Renumber the medical record number in sequence, and the patients were divided into two groups using the random number sequence method. Of the initial cohort of 113 patients, 57 were included in the observation group, and were administered polyethylene glycol 4000 (manufactured by Beau four Ipsen Industry, France, national medicine standard: H20130145) $10 \mathrm{~g}$, twice daily (taken orally). The remaining 56 patients were included in the control group, and were administered lactulose (manufactured by Abbott Biologicals BV, Netherlands, national medicine standard) Word: H20120387) $15 \mathrm{~mL}$, twice daily (taken orally). Patients were given a 3-week course of treatment and were observed for any adverse drug reactions. The clinical effects were compared every week during the 3-week treatment.

There were no statistically significant differences in the general clinical data of patients, including average age, weight, average course of disease, and severity of illness $(\mathrm{P}>0.05)$. None of the enrolled patients suffered from any of the following conditions: severe heart or liver disease, renal dysfunction, cerebrovascular disease caused by paralysis, bedridden, severe endocrine or metabolic disease, colon or rectal cancer, secondary constipation caused by drug-related factors, or allergy to therapeutic drugs. Informed consent was obtained from all patients

Enrolled patients ceased laxatives after admission. Both groups received comprehensive treatments including reasonable diet and appropriate exercise therapy, establishment of a suitable schedule and regular bowel habits, psychological counseling, hot spring baths, massage physiotherapy, and micro-ecological preparations.

This study was conducted in accordance with the ethical principles of the World Medical Association's Declaration of Helsinki (as revised in 2013) and was approved by the ethics committee of the Affiliated Hospital of Jiangnan University [(2017) KY068].

\section{Inclusion and exclusion criteria}

Inclusion criteria: (I) patients with functional constipation according to the Rome IV diagnostic criteria (20) were enrolled in the study. Furthermore, patients meeting two of the following six items were also included: (i) straining for $\geq 25 \%$ of defecation attempts (ii) sensation of incomplete defecation for $\geq 25 \%$ of defecation attempts (iii) lumpy or hard stool for $\geq 25 \%$ of defecation attempts (iv) anorectal obstruction or blockage $\geq 25 \%$ of defecation attempts (v) manual maneuvering required to defecate for $\geq 25 \%$ of defecation attempts (vi) defecation frequency $<3$ times/ week; (II) patients with a single pregnancy, and fetal growth and development in line with the gestational age; (III) patients with good compliance and ability to cooperate for the duration of the study; (IV) patients that signed the informed consent form.

Exclusion criteria: (I) patients with severe pregnancy complications; (II) patients with abnormal gastrointestinal motility caused by other organic or endocrine diseases; (III) patients with severe diseases to the heart, liver, lung, kidney, or other important organs; (IV) patients who had taken relevant treatment drugs within 7 days of the trial; (V) patients who are allergic to research drugs; (VI) patients who often suffered from constipation (before pregnancy); (VII) patients with other digestive system diseases such as intestinal obstruction and inflammatory bowel disease.

\section{Evaluation criteria}

The Wexner constipation scoring system was used to assess the extent of constipation, including (but not limited to) the number and difficulty of bowel movements, degree of emptying, pain, defecation time, assisted defecation, number of defecation failures, and duration of disease. Patients were scored between 0 and 30 points, with a higher score indicating a more serious condition. Moreover, using the Bristol Stool Form Scale (BSFS), patients were given a stool trait score between 1 and 7 to classify their stool form. According to the scale, a score of 1 denotes a stool that is separate hard lumps, indicating severe constipation, while a score of 7 signifies a stool with a liquid consistency and no solid pieces, indicating severe diarrhea. Adverse reactions in 
Table 1 General clinical information

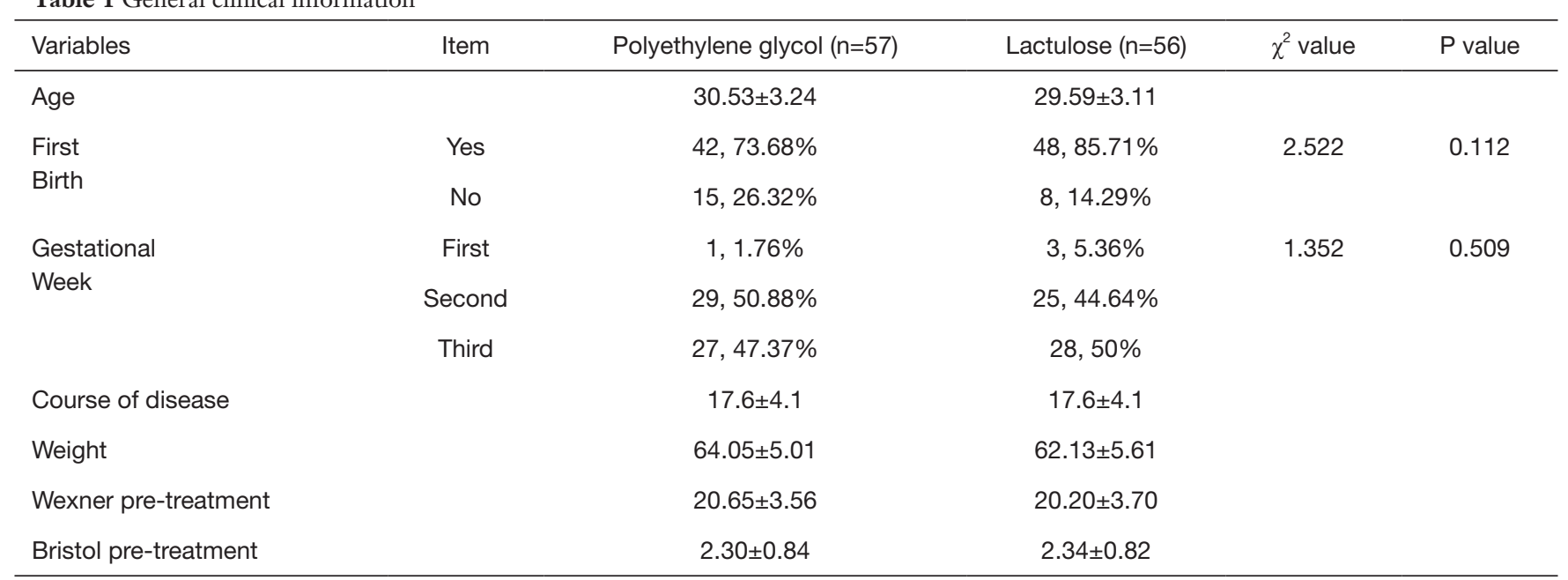

the two groups were recorded, including abdominal pain, diarrhea, allergies, and vomiting.

\section{Statistical analysis}

Data were entered into a spreadsheet (Excel; Microsoft China Co., Ltd., Beijing, China) and SPSS was used for data analysis (V.22.0; SPSS Inc., Chicago, IL, USA). Counting data was compared using the Chi-square $\left(\chi^{2}\right)$ test. Measurement data is shown by $\mu \pm s$. An independent $t$ test was performed for comparison between the groups, and a paired $t$-test was used for intra-group comparison. The Wilcoxon rank-sum test was used to compare the scores of the constipation assessment scale. The data are expressed as mean \pm quartile deviation in box plots and whisker plots. A $\mathrm{P}$ value $<0.05$ was considered statistically significant.

\section{Results}

\section{General clinical characteristics}

In total, 113 pregnant women with constipation were enrolled in this study. Of these, 57 patients were placed into the polyethylene glycol group, and were administered polyethylene glycol 4000 (France Beau four Ipsen Industry Production, National Medicine Standard: H20130145), 10 g, twice daily (taken orally). The patient characteristics in this group were as follows: an average age of $(30.53 \pm 3.24)$ years, primipara $(42,73.68 \%)$, second parturient $(15,26.32 \%)$, average duration of disease $(17.6 \pm 4.1)$ days, average weight $(64.05 \pm 5.01) \mathrm{kg}$, first trimester $(1,1.76 \%)$, second trimester
$(29,50.88 \%)$, third trimester $(27,47.37 \%)$, average Wexner total score before treatment $(20.65 \pm 3.56)$ points, and the average BSFS total score before treatment $(2.30 \pm 0.84)$ points.

The remaining 56 patients were placed into the lactulose (control) group, and were administered lactulose (manufactured by Abbott Biologicals BV, Netherlands, national medicine standard) Word: H20120387), $15 \mathrm{~mL}$, twice daily (taken orally). The patient characteristics in this group were as follows: an average age of $(29.59 \pm 3.11)$ years, primiparas $(48,85.71 \%)$, second parturient $(8,14.29 \%)$, average duration of disease (17.6 \pm 4.1$)$ days, average weight $(62.13 \pm 5.61) \mathrm{kg}$, first trimester $(3,5.36 \%)$, second trimester $(25,44.64 \%)$, third trimester $(28,50 \%)$, average Wexner total score before treatment $(20.20 \pm 3.70)$, and the average BSFS total score before treatment $(2.34 \pm 0.82)$. There were no statistically significant differences between the groups ( $\mathrm{P}>0.05$, Table 1).

\section{Wexner score}

Following the 3-week course of treatment, the two groups were compared. We found that both polyethylene glycol and lactulose were effective in the treatment of constipation $(\mathrm{P}<0.05)$, and there were no significant differences in the total effective rate between the two groups $(\mathrm{P}>0.05)$. However, following our analysis of the scores in the first and second weeks of treatment, we found that the polyethylene glycol group had a faster therapeutic effect $(\mathrm{P}=0.06, \mathrm{P}=0.029$, Table 2). 
Table 2 Comparison of Wexner and BSFS scores between the two groups (n, \%)

\begin{tabular}{lccccc}
\hline \multirow{2}{*}{ Course } & \multicolumn{2}{c}{$\begin{array}{c}\text { Polyethylene glycol } \\
(n=57)\end{array}$} & & \multicolumn{2}{c}{$\begin{array}{c}\text { Lactulose } \\
(\mathrm{n}=56)\end{array}$} \\
\cline { 2 - 3 } \cline { 5 - 6 } & Wexner & BSFS & & Wexner & BSFS \\
\hline Pre-treatment & $20.65 \pm 3.56$ & $2.30 \pm 0.84$ & & $20.20 \pm 3.70$ & $2.34 \pm 0.82$ \\
1 week & $12.51 \pm 4.05^{\mathrm{a}}$ & $2.81 \pm 0.79$ & & $13.96 \pm 4.46$ & $2.66 \pm 0.72$ \\
2 weeks & $8.54 \pm 3.06^{\mathrm{b}}$ & $3.21 \pm 0.79$ & & $10.00 \pm 3.73$ & $3.20 \pm 0.73$ \\
3 weeks & $5.74 \pm 1.16^{c}$ & $3.91 \pm 0.79^{c}$ & & $5.91 \pm 1.35^{c}$ & $3.77 \pm 0.69^{c}$ \\
\hline
\end{tabular}

a,$P=0.06$ compared to the first week of the Lactulose treatment group; ${ }^{b}, P=0.029$ compared to the second week of the Lactulose treatment group; ${ }^{c}, \mathrm{P}<0.05$ compared to pre-treatment. BSFS, Bristol Stool Form Scale.
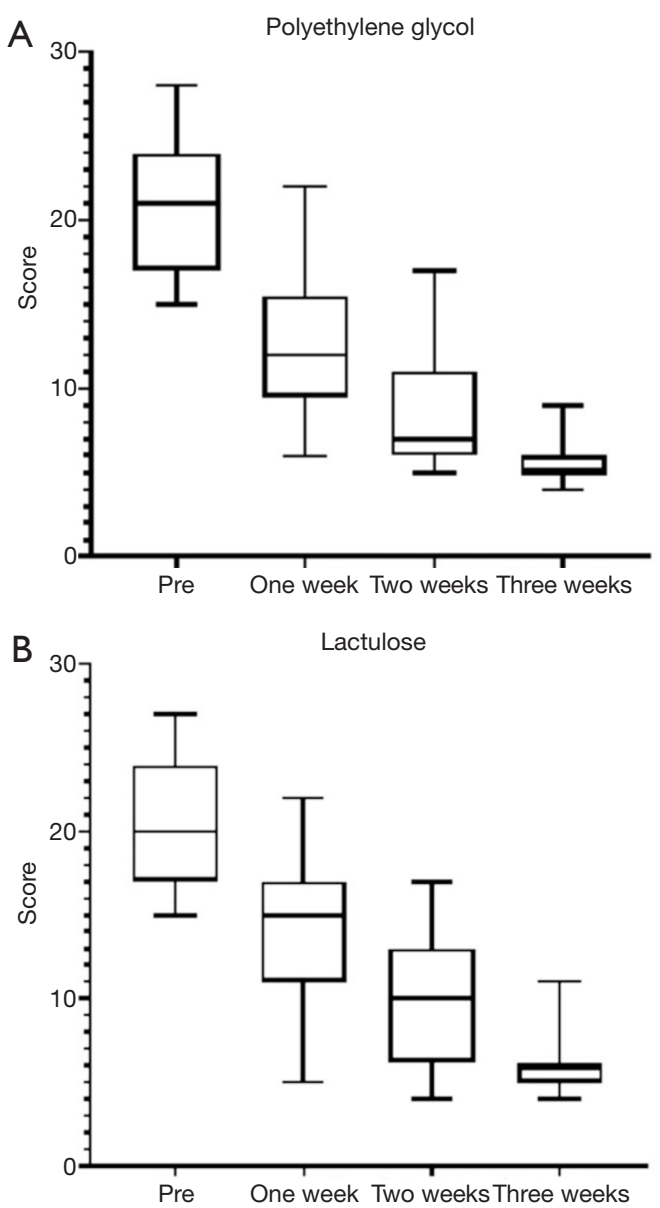

Figure 1 Wexner scores before and after treatment.

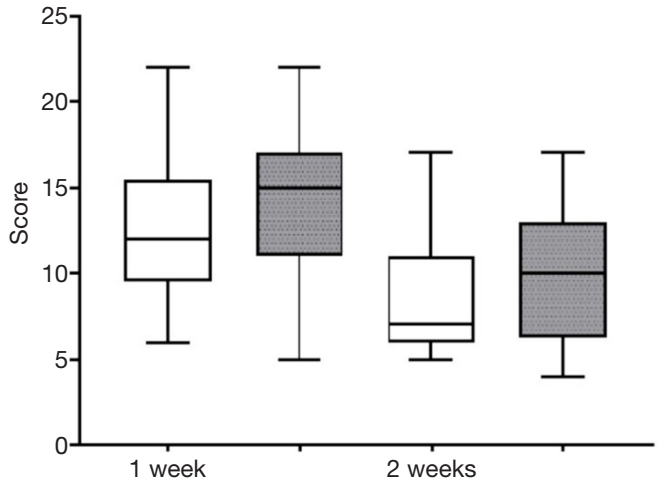

Figure 2 Comparison of Polyethylene glycol and Lactulose scores between the two groups (after 1 week and after 2 weeks).

\section{Bristol stool form scale score}

According to the BSFS scores, both groups were effective $(\mathrm{P}<0.05)$ and there was no significant difference in the total effective rate between the groups $(\mathrm{P}>0.05$, Table 2, Figures 1,2).

\section{Adverse reactions}

In the polyethylene glycol group, three patients had abdominal pain $(5.3 \%)$, one patient had diarrhea $(1.8 \%)$, one patient experienced vomiting $(1.8 \%)$, and none of the patients experienced an allergic reaction $(0 \%)$. For the lactulose group, two patients had abdominal pain (3.6\%), two patients had diarrhea (3.6\%), one patient experienced vomiting $(1.8 \%)$, and none of the patients experienced an allergic reaction $(0 \%)$. Furthermore, there was no discontinuation of treatment due to adverse reactions throughout the study period, and no abnormalities in the fetus were found during follow-up. These results indicate that there was no significant difference in the incidence of adverse reactions between the two groups (Table 3).

\section{Discussion}

Owing to the particular physiology of pregnancy, the incidence of constipation in pregnant women is higher compared to the general population, and there are fewer therapeutic drugs to choose from. Generally, as the gestational age of the pregnancy increases, so do the 
Table 3 Comparison of adverse reactions between the two groups (n, \%)

\begin{tabular}{lcccc}
\hline Group & Abdominal pain & Diarrhea & Allergy & Vomiting \\
\hline $\begin{array}{l}\text { Polyethylene glycol } \\
(\mathrm{n}=57)\end{array}$ & $3(5.3)$ & $1(1.8)$ & $0(0)$ & $1(1.8)$ \\
Lactulose $(\mathrm{n}=56)$ & $2(3.6)$ & $2(3.6)$ & $0(0)$ & $1(1.8)$ \\
\hline
\end{tabular}

levels of progesterone, resulting in slower gastrointestinal peristalsis and weakened tension. This is coupled with the increased pressure of the uterus on the gastrointestinal tract, and the reduced peristaltic function of the rectum. These changes are not conducive to the movement of stool to the anus, and thus, the stool remains in the rectum for an extended period. Furthermore, a considerable amount of water can be absorbed in the intestine, resulting in the stool becoming a hard mass that is difficult to excrete and can easily lead to constipation (21). Psychological and dietary interventions are typically recommended for newonset constipation in pregnant patients, and medications can be considered where these therapies prove ineffective. The formulation of safe and effective drug treatment plans has important clinical significance for the safety of both mothers and babies.

Lactulose is a commonly used drug for the clinical treatment of functional constipation and has little effect on the fetus. Under the action of osmotic pressure, it promotes the softening of feces, increases the volume of the intestinal cavity, promotes the peristalsis of the intestine, and ultimately assists in the excretion of feces (9). It can also increase the number of intestinal probiotics such as Lactobacillus and Bifidobacterium, reduce the number of anaerobic bacteria, and regulate the micro-ecological balance of the intestinal tract (10). Polyethylene glycol 4000 powder is a high-molecular weight polymer. Upon entering the intestinal tract, it increases the water content of feces and promotes its movement through the intestinal tract to be excreted from the body (22). Polyethylene glycol is not metabolized or absorbed in the intestine. Also, it does not affect the absorption of electrolytes, fat-soluble vitamins, and other nutrients, and does not impact colonic transit time or motility. Neither of the two drugs will be absorbed into the blood through the intestinal tract, which will not cause significant impact on the mother and fetus, and the treatment is relatively safe.

In this study, the total effective rate increased after treatment using both medications. Analysis of the scores in the first and second week between the polyethylene glycol group and the lactulose group shows that the former has a more rapid therapeutic effect than the latter $(\mathrm{P}=0.06$, $\mathrm{P}=0.029$ ), and can effectively shorten the duration of the disease. Considering that there were no differences in the incidence of adverse reactions following treatment, we suggest that neither polyethylene glycol nor lactulose increases the risk of adverse reactions. Both drugs were not absorbed into the blood through the intestine, and did not lead to significant effects on the mother or the fetus.

Clinical treatment of constipation should not only take into account physiological function, but also the quality of life of the patient. Treatment of constipation can cause significant disruption to quality of life and seriously impact the physical and mental health of pregnant women, and can also lead to adverse risks to the development of the fetus. Thus, improving the quality of life of patients with constipation during pregnancy has crucial clinical significance for ensuring that the pregnancy is safe and for protecting the healthy development of fetuses.

In summary, polyethylene glycol and lactulose can both effectively treat constipation during pregnancy and improve the quality of life of patients. Patients using polyethylene glycol experience faster relief of clinical symptoms and shorter disease duration. However, the number of cases included in this experiment is limited, and the results may be biased. More research in this area is needed in the future to confirm our findings.

\section{Acknowledgments}

Funding: (I) Top Talents Project of "Six-one Project" for High-level Health Talents in Jiangsu Province (LGY2018016); (II) Key Talents Project of "Strengthening Health through Science and Education" of Wuxi Health and Family Planning Commission (ZDRC039); (III) Application and Translation of Key Techniques on Gastrointestinal Lubricant (ZM008).

\section{Footnote}

Reporting Checklist: The authors have completed the CONSORT reporting checklist. Available at http://dx.doi. org/10.21037/apm-20-1674

Data Sharing Statement: Available at http://dx.doi. org/10.21037/apm-20-1674 
Conflicts of Interest: All authors have completed the ICMJE uniform disclosure form (available at http://dx.doi. org/10.21037/apm-20-1674). The authors have no conflicts of interest to declare.

Ethical Statement: The authors are accountable for all aspects of the work in ensuring that questions related to the accuracy or integrity of any part of the work are appropriately investigated and resolved. This study was conducted in accordance with the ethical principles of the World Medical Association's Declaration of Helsinki (as revised in 2013) and was approved by the ethics committee of the Affiliated Hospital of Jiangnan University [(2017) KY068]. Informed consent was obtained from all patients.

Open Access Statement: This is an Open Access article distributed in accordance with the Creative Commons Attribution-NonCommercial-NoDerivs 4.0 International License (CC BY-NC-ND 4.0), which permits the noncommercial replication and distribution of the article with the strict proviso that no changes or edits are made and the original work is properly cited (including links to both the formal publication through the relevant DOI and the license). See: https://creativecommons.org/licenses/by-nc-nd/4.0/.

\section{References}

1. Chu H, Zhong L, Li H, et al. Epidemiology characteristics of constipation for general population, pediatric population, and elderly population in china. Gastroenterol Res Pract 2014;2014:532734.

2. Cullen G, O'Donoghue D. Constipation and pregnancy. Best Pract Res Clin Gastroenterol 2007;21:807-18.

3. Mulak A, Tache Y, Larauche M. Sex hormones in the modulation of irritable bowel syndrome. World J Gastroenterol 2014;20:2433-48.

4. Trottier M, Erebara A, Bozzo P. Treating constipation during pregnancy. Can Fam Physician 2012;58:836-8.

5. Pillitteri A. Maternal and child health nursing: Care of the childbearing and childrearing family. 6 ed. Lippincott Williams and Wilkins; 2010.

6. Slavin JL. Position of the American Dietetic Association: health implications of dietary fiber. J Am Diet Assoc 2008;108:1716-31.

7. American College of Gastroenterology Chronic Constipation Task Force. An evidence-based approach to the management of chronic constipation in North America. Am J Gastroenterol 2005;100 Suppl 1:S1-4.
8. Mahadevan U, Kane S. American gastroenterological association institute technical review on the use of gastrointestinal medications in pregnancy. Gastroenterology 2006;131:283-311.

9. Liang SH, Wen P, Ye XP et al. A preliminary study on the treatment of constipation during pregnancy with lactulose supplemented with moderate activity. Chinese Journal of Disease Control and Prevention 2009;13:598-9.

10. Lactulose Clinical Cooperation Group, Zhu ZZ. A randomized, double-blind, placebo-controlled multicenter clinical study of lactulose in the treatment of constipation in pregnant women. Chinese Journal of Digestion 2006;(10):690-3.

11. Lachgar M, Morer I. Efficiency and tolerance of lactulose in constipation in pregnant women. Rev Fr Gynecol Obstet 1985;80:663-5.

12. Müller M, Jaquenoud E. Treatment of constipation in pregnant women. A multicenter study in a gynecological practice. Schweiz Med Wochenschr 1995;125:1689-93.

13. Mahony R, Behan M, O'Herlihy C, et al. Randomized, clinical trial of bowel confinement vs. laxative use after primary repair of a third-degree obstetric anal sphincter tear. Dis Colon Rectum 2004;47:12-7.

14. Eogan M, Daly L, Behan M, et al. Randomised clinical trial of a laxative alone versus a laxative and a bulking agent after primary repair of obstetric anal sphincter injury. BJOG 2007;114:736-40.

15. Ferchaud-Roucher V, Pouteau E, Piloquet H, et al. Colonic fermentation from lactulose inhibits lipolysis in overweight subjects. Am J Physiol Endocrinol Metab 2005;289:E716-20.

16. Herve S, Leroi AM, Mathiex-Fortunet $\mathrm{H}$, et al. Effects of polyethylene glycol 4000 on 24-h manometric recordings of left colonic motor activity. Eur J Gastroenterol Hepatol 2001;13:647-54.

17. Zhou LY, Xia ZW, Lin SR, et al. Multicenter randomized controlled clinical trial study of polyethylene glycol 4000 in the treatment of adult chronic functional constipation. Chinese Journal of Clinical Pharmacology 2001;17:7-10.

18. Ford AC, Moayyedi P, Lacy BE, et al. American College of Gastroenterology monograph on the management of irritable bowel syndrome and chronic idiopathic constipation. Am J Gastroenterol 2014;109 Suppl 1:S2-26; quiz S27.

19. Treepongkaruna S, Simakachorn N, Pienvichit P, et al. A randomised, double-blind study of polyethylene glycol 4000 and lactulose in the treatment of constipation in children. BMC Pediatr 2014;14:153. 
20. Li JX, Chen X, Ke X. Opinions on the combined diagnosis and treatment of Chinese and Western medicine for functional constipation (2017). Chinese Journal of Integrated Traditional Chinese and Western Medicine on Digestion 2018;26:18.

21. Shi WJ, Ma W, Xiang XM et al. Investigation and analysis of functional constipation in pregnant women. Journal of
Practical Hospital Clinic 2017;14:52-5.

22. Zhang SF, Liu W Zhou PP, et al. Study on the influence of different polyethylene glycol 4000 powder prescriptions on intestinal cleanliness and safety evaluation. Zhongqing Medical Science 2017;46:1492-4.

(English Language Editor: A. Kassem)
Cite this article as: $\mathrm{Li} \mathrm{H}$, Zhang P, Xue Y. A comparison of the safety and efficacy of polyethylene glycol 4000 and lactulose for the treatment of constipation in pregnant women: a randomized controlled clinical study. Ann Palliat Med 2020;9(6):3785-3792. doi: 10.21037/apm-20-1674 